\title{
Transhumanismus im Kreuzfeuer der Theologie? Eine Analyse der theologischen Kritik am Transhumanismus
}

\section{Einleitung}

Dieser Aufsatz untersucht die grundsätzlichen Herausforderungen, mit denen die ethische Reflexion vor allem im Bereich der Theologie durch den Transhumanismus ${ }^{2}$ (TH) konfrontiert ist. Man wird sich vielleicht fragen, was der $\mathrm{TH}$ in einem Band über Digitalisierung soll. Weil eine der neusten Entwicklungen digitaler Technologie, Artificial Intelligence (AI), neben Gen- und Nanotechnologie zugleich auch einer der zentralen Treiber des Transhumanismus ist (Bostrom 2014), ist es durchaus sinnvoll, das Thema in diesem Kontext aufzunehmen. Wer über Chancen und Risiken der digitalen Gesellschaft spricht, wird eher früher als später auch auf das Konzept des Transhumanismus stoßen.

Was ist Transhumanismus? Dazu ein Zitat von Max More (1990), einem der Begründer des TH:

„Transhumanism is a class of philosophies that seek to guide us towards a posthuman condition. Transhumanism shares many elements of humanism, including a respect for reason and science, a commitment to progress, and a valuing of human (or transhuman) existence in this life rather than in some supernatural 'afterlife'. Transhumanism differs from humanism in recognizing and anticipating the radical alterations in the nature and possibilities of our lives resulting from various sciences and technologies such as neuroscience and neuropharmacology, life extension, nanotechnology, artificial ultraintelligence, and space habitation, combined with a rational philosophy and value system."

Janina Loh (2019a) hat in ihrer Einführung zwischen Transhumanismus (der den Menschen verbessern will), technologischem Posthumanismus (der eine IT-basierte Superintelligenz schaffen will) und kritischem Posthumanismus (der das humanistische Verständnis des Menschen überwinden will) unterschieden (vgl. ebd., 14). Die entsprechenden Autor*innen selbst

1 Ich danke Barbara Bleisch, Peter Kirchschläger, Birte Platow und Gotlind Ulshöfer für ihre äußerst hilfreichen Kommentare zu meinen Überlegungen.

2 Im Folgenden als TH abgekürzt. Ich kürze auch den Ausdruck „Transhumanist*innen“ mit TH ab. Was gemeint ist, erschließt sich jeweils problemlos aus dem Kontext. 
machen diese im Hinblick auf eine systematische Darstellung gemachte begriffliche Unterscheidung natürlich nicht unbedingt. Meine Überlegungen gehen zwar im Wesentlichen vom TH im Sinne von Loh (2019a) aus, aber auch die beiden anderen Aspekte spielen eine, wenn auch kleine, Rolle. Der Einfachheit halber fasse ich alles unter dem Label Transhumanismus zusammen.

Im Hinblick auf die folgende Diskussion reichen m. E. die drei folgenden, charakterisierenden Merkmale des TH:

1. Der TH beurteilt die Endlichkeit und Unvollkommenheit menschlichen Lebens und der damit verbundenen Leiden und Ineffizienzen ausschließlich negativ.

2. Die negativen Einschränkungen menschlichen Lebens sollen nach Möglichkeit aufgehoben werden. Das gegenwärtige biologisch-körperliche Substrat des Menschen soll dabei radikal transformiert werden, in einigen Versionen sogar überwunden werden. ${ }^{3}$

3. Ziel ist im Vergleich zum heutigen ein in vielerlei Hinsicht „besseres“ Leben („human flourishing“), und vor allem auch ein viel längeres Leben. ${ }^{4}$

Einige interpretieren dies alles so, dass im Rahmen des TH Unsterblichkeit, Glück und Göttlichkeit des Menschen angestrebt werden (Harari 2015), andere sprechen davon, dass „Leiblich-Kontingentes“ im TH ein „zu überwindender Störfaktor" sei, und dass es im TH letztlich um Kontingenzbeseitigung gehe (Klöcker 2018, 332 f.). ${ }^{5}$

Von Seiten der Theologie wird der TH fast ausschließlich negativ beurteilt: Kategorien wie Sünde, Hybris oder Idolatrie sind schnell zur Hand (Peters 2011; Child 2015). Das ist nichts Neues, sondern steht in einer Linie mit den theologischen Reaktionen die vor 20 Jahren Peter Sloterdijks „Regeln für den Menschenpark“ (1999) ausgelöst hatten. Insbesondere im deutschsprachigen Raum sind Theolog*innen mit wenigen Ausnahmen (Wirth 2018) recht hilflos, wenn es um die Fragen einer grundsätzlichen Beurteilung des TH geht. Im Grundsatz scheinen sich viele Theolog*innen

3 Vgl. dazu den signifikanten Titel von Kurzweil 2005 "When humans transcend biology".

4 Gemäss Child 2015 gehören zur transhumanen Zukunft "development of superintelligent machines"; "lifelong emotional well-being"; "vastly extended life"; "uploading one's consciousness" $(2015,9)$.

5 Spieckermann 2019 hat in diesem Zusammenhang von den „lieblosen Ideen der Transhumanisten“ gesprochen: „Im Transhumanismus verdichtet sich das negative Menschenbild $\mathrm{zu}$ einer Ideologie der Lieblosigkeit, in der der Mensch schlichtweg als suboptimales Auslaufmodell angesehen wird." (Spieckermann 2019, 166). 
einig: Im TH werden, wie es Childs 2015 besonders deutlich ausführt, dem Menschen vorgegebene Grenzen überschritten.

Mein Aufsatz diskutiert die mit diesem Vorwurf gegebenen Probleme und Strategien kritisch. Ich zeige im zentralen Abschnitt 2, dass theologischen Argumenten gegen den TH oft eine essentialistische oder biokonservative Tendenz zugrunde liegt, und dass diese Tendenz drei Probleme mit sich bringt, welche die durch den $\mathrm{TH}$ induzierten Veränderungen und Herausforderungen unserer Moral mit ihrem Fokus auf Menschenwürde oder Menschenrechten nicht adäquat wahrnehmen. Der Reihe nach diskutiere ich zunächst ausführlich das Problem der „Abschaffung des Menschen“ (Problem 1). Darauf folgen mit „die unscharfen Grenzen zwischen menschlichen und nicht-menschlichen Entiäten“ (Problem 2) und „Grenzen des technologischen Fortschrittes“ (Problem 3) zwei weitere Probleme. Problem 1 nehme ich auf, weil die grundsätzlichste theologische Kritik am TH die ist, dass er das, was den Menschen ausmacht und wertvoll macht, abschaffen werde. Problem 2 folgt direkt aus meiner Diskussion von Problem 1 und mit Problem 3 soll abschließend ein typisches Thema der angewandten Ethik etwas grundsätzlicher beleuchtet werden. Alle theologischen Positionen, die ich aufgreifen werde, haben mögliche Einwände gegen sie selbstverständlich diskutiert und ihre Argumente verfeinert. Auf diese Debatte kann aber im Einzelnen nicht eingegangen werden. Es geht mir in diesem Aufsatz nur darum, einige theologische Einwände gegen den TH und damit verbundene Probleme kritisch zu beleuchten. Im abschließenden Teil (Abschnitt 3) des Aufsatzes zeige ich, wie die grundsätzliche Beurteilung des TH meines Erachtens ansetzen müsste, und inwiefern der TH auch der Theologie zu denken geben kann.

Dieser Aufsatz untersucht nicht die Frage des Realitätsgehaltes der Prognosen bezüglich TH und AI wie sie bspw. Kurzweil 2005 vorlegt (vgl. Klaes 2019). Auch kein Thema sind die möglichen oder wahrscheinlichen Kosten und Nutzen von transhumanistischen Konzepten und Technologien sowie deren ethische Bewertung bspw. Gerechtigkeitsfragen im Hinblick auf eine mögliche Zweiklassenmenschheit (vgl. Heilinger 2016). Der Fokus meines Aufsatzes liegt auch nicht auf den spezifischen Schwächen der THPositionen. Mich interessiert ausschließlich die oft sehr fundamental-kritische und zugleich defensive Reaktion der Theologie auf den TH und die 
mit ihm gegebenen Herausforderung bezüglich einiger unser moralischen Grundkonzepte wie Menschenwürde und Menschenrechte. ${ }^{6}$

\section{Argumente gegen die theologische Kritik am Transhumanismus}

Im Hinblick auf eine Diskussion der theologisch monierten Grenzüberschreitung durch den TH scheinen mir besonders zwei Argumentationsstränge relevant.

Der TH führe erstens zu einer „Überschreitung der Natur des Menschen" (Klöcker 2018, 318). Die Vermutung liegt nahe, dass hier eine essentialistisch-biokonservative Sichtweise ins Spiel kommt. Erwähnt sei etwa das (neo-thomistische) Festhalten an so etwas wie „human nature“verbunden mit einer Vorstellung eines „ultimate good“ des Menschen durch Gerald McKenny (2011, 179ff und 185 ff.). Ein analoges Beispiel: James Childs Jr. nimmt zustimmend Bezug auf McKennys These, dass im Falle des TH von menschlicher Aktivität gesprochen werden könne, die keinen Bezug auf „human nature" habe. Dies führe, so Childs im Anschluss an Alasdair MacIntyre, zu einem „loss of telos“ und damit zu einem Verlust der Möglichkeit von Ethik überhaupt (Childs 2015, 13f.). Gemeint ist damit der Verlust einer an eine Konzeption von menschlicher Natur gebundene objektive Konzeption von menschlicher Erfüllung („human fulfillment“). ${ }^{7}$ Auch Ulrich Körtner $(2013,209)$ hält an einer ,final determination ... of the human being" fest. Bei ihm ist, typisch für theologische Positionen, die mit dieser finalen Bestimmung des Menschen anvisierte „perfection“ etwas, was nicht in den Aufgabenbereich des Menschen, sondern Gottes gehört. Oder mit den Worten von McKenny $(2011,185):$,... it is the gift of divine grace that extends them [human capacities], and not a technological enhancement."

Um damit zum zweiten der oben in Aussicht gestellten Argumentationsstränge zu kommen. Es komme zur Aufhebung dessen, was Autonomie ermöglicht, d. h. der „Konstitutionsbedingungen von Autonomie“ (Klöcker

6 Im Folgenden finden sich zahlreiche Fußnoten. Der oder die eilige Leser*in kann diese getrost überspringen. Die Argumentation erschließt sich auch ohne sie. Wer wissen will, welche Texte mich zusätzlich zu den im fortlaufenden Text genannten angeregt haben, findet in den Fußnoten weiterführende Angaben und Bemerkungen.

7 Auch TH haben natürlich eine Vorstellung von menschlicher Erfüllung, aber - darin hat Childs Recht - sie ist nicht an ein objektives Telos gebunden, sondern an subjektive Präferenzen von Individuen: "subjective choice replaces any sense of a teleological standard for human fulfillment. It is the height of unrestrained autonomy in which moral authority seems unnecessary" (Childs 2015, 14). 
2018, 331) und damit der autonomen Person. Gerade in seiner Kreativität werde der Mensch ja damit konfrontiert, „dass sich Bereiche seines Lebens der Machbarkeit und Gestaltbarkeit entziehen" (Klöcker 2018, 328). Zu den erwähnten Konstitutionsbedingungen gehören „die Begrenztheit des Menschen, seine Endlichkeit, seine Verletzbarkeit" (2018, 331). Damit muss sicher, wenn auch vielleicht nicht ausschließlich, die biologische Grundausstattung des Menschen und die darauf basierende Verletzbarkeit und Endlichkeit (bis hin zum Tod) gemeint sein. Moralisch legitim seien vor diesem Hintergrund nur Optimierungsziele und Enhancement-Bestrebungen „die einen in dieser Weise umfassenden Begriff von Autonomie sichern" $(2018$, 332). Wer, wie im Falle des TH, die Autonomie des Menschen (also die Bedingung der Möglichkeit von Selbst- und Weltgestaltung) einsetzt, um die gegebene Biologie des Menschen zu überwinden, betritt verbotenes Gelände. Denn damit verschwinde der Grund von und für eine humane Ethik (Klöcker 2018, 335). Das Thema der Autonomie hatte seinerzeit ja schon Habermas (allerdings besonders im Hinblick auf Keimbahninterventionen und vorgeburtliche Selektion) beschäftigt: Es gehe um ein Menschsein, indem „wir uns weiterhin als ungeteilte Autoren unserer Lebensgeschichte verstehen werden und uns gegenseitig als autonom handelnde Personen anerkennen können“ (Habermas 2001, 49). Er sieht dabei insbesondere die Gefahr einer „Selbstbemächtigung“, die die „notwendigen Bedingungen für autonome Lebensführung und ein universalistisches Verständnis von Moral berühren könnte" $(2001,85)$.

Das theologische Urteil von heute ist klar und scharf: Dieses Operieren an den Konstitutionsbedingungen menschlicher Autonomie darf nicht sein. Es kommt einer Abschaffung des Menschen, wie er gegeben ist, gleich. ${ }^{8}$

\subsection{Die Abschaffung des Menschen (Problem 1)}

Die von Theolog*innen formulierte ethische Kritik des TH lebt von starken Voraussetzungen. Es kommt wie eben gesehen explizit oder implizit zu einem Rückgriff auf so etwas wie „die Natur des Menschen“, oder etwas vorsichtiger formuliert „das, was den Menschen ausmacht“. Katharina Klöcker $(2018$, 329) ist der Ansicht, es könne dabei von einer essentialistischen Engführung im Falle ihrer theologischen Kritik am TH nicht die Rede

8 Die Gründer der Christian Transhumanist Association, Christopher Benek und Micah Redding sehen das natürlich anders. Vgl. dazu Thweatt 2018, 368 ff. 
sein. ${ }^{9}$ Ihr Argument, aufgrund der geschichtlichen Offenheit des Menschen lasse sich ein „statischer und zeitlos gültiger Begriff vom Menschen“ nicht konstruieren, ist allerdings nicht konsistent. De facto knüpft auch sie ihren normativen Begriff der Autonomie an gegebene, leiblich-kontingente Wesensmerkmale des Menschen (2018, 331 f.). Was aber viel entscheidender ist: Dem, was hier vorausgesetzt wird, wird von den Theolog*innen ein universaler moralischer Wert zugesprochen. ${ }^{10}$

Was heißt das? Zunächst einmal behauptet in diesem Kontext niemand, die Biologie des Menschen sei es, was die "Natur" des Menschen exklusiv bestimme. Aber dennoch gilt der (!) Mensch als Entität mit einer spezifischen, biologische-endlichen Ausstattung (und der damit gegebenen Begrenztheit, Endlichkeit und Verletzbarkeit) im in Anspruch genommenen axiologischen Rahmen als unverfügbar im Sinne von „moralisch unbedingt schützenswert“. Geläufige Konzepte wie die Menschenwürde oder die Menschenrechte bringen entsprechende Schutzvorstellungen zur Sprache (Körtner 2013, 203 ff.). Menschenwürde- und Menschenrechtsverletzungen sind in diesem anthropologischen Rahmen nicht abtrennbar verbunden mit Verletzungen von so etwas wie "menschlicher Natur“. ${ }^{11}$ Das Anwendungsgebiet der grundlegenden normativen Konzeptionen „Menschenwürde und Menschenrechte" ist der Mensch als ein biologisch-natürlich entstandenes und konstituiertes endliches Wesen. ${ }^{12}$

Dieses Verständnis vom Menschen übernehmen die Vertreter*innen des TH bekanntlich nicht. Aus der Tatsache, dass biologisch-endliche Menschen formbar und veränderbar, also nicht ein für alle Mal festgelegt sind, schließen sie vielmehr, dass diese im Hinblick auf bestimmte Ziele wie Glück, Wohlergehen oder sehr langes Leben transformiert werden dürfen. Wenn ich es recht sehe, sind mit dem Formulieren derartiger Ziele keine Aussagen über eine feststehende Natur des Menschen verbunden. Es geht um die Erfüllung spezifischer Präferenzen, die Menschen heute in aller Regel haben, ohne dass diese Präferenzen auch in Zukunft vorliegen

9 Klöcker definiert „essentialistische Engführung“ etwas enigmatisch im Sinne einer „Deduktion normativer Vorgaben behaupteter Wesenseigenschaften“ $(2018,329)$. Will sie damit Essentialismus als einen Sein-Sollen-Fehlschluss thematisieren?

$10 \mathrm{Zu}$ diesem Thema vgl. Zimmermann 2015, 185.

$11 \mathrm{Ob}$ der Menschenrechtsdiskurs generell einen derartigen anthropologischen Rahmen voraussetzt, respektive in der Diktion von Peter Kirchschläger: ein Menschenbild benötigt, ist eine andere Frage (vgl. dazu Kirchschläger 2015). Mir geht es hier nur um die Auseinandersetzung mit dem TH.

12 Damit nehme ich die beiden Aspekte von Natürlichkeit, „genetische“ und „qualitative“, gemäß Birnbacher 2006, 7 ff. auf. 
müssten. Es ist gar nicht so einfach, gegen derartige Enhancement-Visionen zu argumentieren: Aus der Tatsache, dass menschliches Leben und Wohlergehen heute an eine bestimmte Biologie gebunden ist, kann ja nicht direkt abgeleitet werden, diese biologische Natur des Menschen sei moralisch schützenswert. Das wäre ein naturalistischer Fehlschluss. ${ }^{13}$ Konsequenterweise ist denn auch bei einem Ethiker wie Peter Singer (2011) nicht der menschliche Körper oder gar so etwas wie eine menschliche Natur moralisch wertvoll und schützenswert. Moralisch relevant sind nur die Präferenzen, welche Menschen als biologische Wesen mit Interessen haben.

Interessant wird diese Debatte, da sich auch die Gegner des TH ja nicht prinzipiell gegen Transformationen des Menschen aussprechen. Treffend bringt dies McKenny $(2011,117)$ zum Ausdruck, der von „legitimacy of some degree of dissatisfaction with the limits of human nature" spricht. Allerdings darf dies zu Transformationen bloß innerhalb bestimmter Grenzen führen. Gemäß Katharina Klöcker (2018) etwa dürfen dabei, wie oben schon ausgeführt, die Autonomie und die sie ermöglichenden natürlichen Konstitutionsbedingungen nicht verletzt werden. Theologisch gesehen liegen zudem mit Visionen vom ewigen Leben Vorstellungen vor, die eine gewisse Ähnlichkeit mit denen des TH haben. Allerdings, dies die unüberschreitbare Grenze jedenfalls im Mainstream, gilt als Subjekt der Realisierung derartiger Visionen dezidiert nicht der Mensch, sondern eben ausschließlich Gott (Körtner 2013, 209 f.). ${ }^{14}$ Ich werde diese theologische Version der erlaubten und möglichen Transformation des Menschen weiter hinten, in Abschnitt 3, explizit aufnehmen.

Wie ist diese Diskussionslage zu interpretieren? M. E. greift die Verwendung der moralischen Begrifflichkeit von Menschenwürde und Menschenrechten gegen den $\mathrm{TH}$ in gewisser Weise ins Leere. Die Bedingungen, von Autonomie (oder Menschenwürde und Menschenrechten) in einem uns vertrauten Sinn zu reden, sind nach den AI/TH-Transformationen unter Umständen gar nicht mehr gegeben. Man kann das auch daran deutlich machen, dass zu den diskutierten transhumanen Entitäten auch superintelligente Artificial Intelligence (AI)-Entitäten gehören würden, die sich der Beurteilung durch das, was Menschen ausmacht, entziehen: „It is important

13 Noch weniger erfolgsversprechend scheinen mir Versuche wie diejenige von Stefan Weit (2018, 226), der seine Kritik am TH auf einer „Metaphysik des Menschen“ aufbaut.

14 "Within Christian theology, the promise of resurrection looks like it will carry us beyond the boundary of current human nature. Yet, we might look at our future resurrection as our human nature, our essential human nature as God creates us" (Childs $2015,15)$. 
not to anthropomorphize superintelligence when thinking about its potential impacts" (Bostrom 2014, 111). TH/AI-Entwicklungen könnten unsere Welt und Gesellschaften grundsätzlich hinsichtlich der moralischen Vorstellungen transformieren, die wir für richtig halten: Es wird klar, so Bostrom \& Yudkowsky (2014, 329), „that superhuman intelligence presents ethical challenges that are quite literally unprecedented." Wie soll man aber mit Vertreter*innen von Positionen diskutieren, die offensichtlich einige grundlegende moralische Intuitionen nicht teilen, die den meisten anderen (Theolog*innen und Philosoph*innen) sehr wichtig sind? Um die vom $\mathrm{TH}$ vorgesehenen Entwicklungen der Kritik unterziehen zu können, muss man voraussetzen, was erst gezeigt werden müsste: dass nämlich die Menschenwürde und Menschenrechte für alle Wesen Gültigkeit haben, die wie auch immer vom jetzigen (biologischen) Menschen „abstammen“, und dass es daher moralisch verboten ist, trans- oder posthumane Wesen zu erzeugen.

Was die Diskussion erschwert: Die von der Theologie der Kritik unterzogenen TH/AI-Positionen verzichten nicht auf die Rede von Menschenwürde oder Menschenrechten und deren normativen Gehalte (vgl. Bostrom 2005), auch wenn sie sich vielleicht bloß in selektiver Weise an ihrem normativen Gehalt orientieren. Oft scheint es eher um andere Interpretationen und Anwendungen der altbekannten Prinzipien bspw. der biomedizinischen Ethik zu gehen:

„The general point here is that when thinking about applied ethics for contexts that are very different from our familiar human condition, we must be careful not to mistake mid-level ethical principles for foundational normative truths. Put differently, we must recognize both the extent to which our ordinary normative precepts are implicitly conditioned on the obtaining of various empirical conditions, and the need to adjust these precepts accordingly when applying them to hypothetical futuristic cases in which their preconditions are assumed not to obtain. By this, we are not making any controversial claim about moral relativism, but merely highlighting the commonsensical point that context is relevant to the application of ethics - and suggesting that this point is especially pertinent when one is considering the ethics of minds with exotic properties." (Bostrom \& Budkowsky 2014, 328)

Um es zu wiederholen: Das Problem scheint dies zu sein, dass die Bedingungen, unter denen wir heute von Autonomie (oder Menschenwürde und Menschenrechten) in einem uns vertrauten Sinn reden können, nach den AI/TH-Transformationen unter Umständen gar nicht mehr gegeben sind (Harari 2017, 206). 
„Do we really want to get to this place?“, fragt Ted Peters $(2011,70)$. Aber weil nicht wir heute Lebenden mit unseren Präferenzen und Überzeugungen es sind, die dann leben würden, müssen wir gemäß Bostrom (2014, 207 f.) der Versuchung widerstehen, unsere eigenen Vorstellungen auf diese Zukunft anzuwenden. In meiner Diagnose haben wir es hier mit einem Spezialfall des von Derek Parfit (1987, 351 ff.) erstmals diskutierten NonIdentity-Problems zu tun. Auch hier geht es um die Frage der menschlichen Zukunft, insbesondere um die Frage, wie wir damit umgehen können, dass unser Wissen bezüglich der in Zukunft lebenden Menschen sehr beschränkt ist: Wir können nicht wissen, welche spezifischen Interessen und Bedürfnisse weit nach uns Lebende haben werden. Deren Bedürfnisse hängen erstens von politischen Entscheiden und gesellschaftlichen Aktivitäten (mit bspw. spezifischen sozialen, ökologischen und wirtschaftlichen Folgen sowie entsprechend angepassten Präferenzen), die erst nach heute erfolgen, und zweitens von den damit verbundenen, zukünftigen politischen, sozialen und ökonomischen Werten und Institutionen ab. Gemäß Parfit kann man sich also bezüglich der Zukunftsverantwortung nicht auf unabhängige Interessen beziehen, die über basale biologische Interessen hinausgehen. Es sind keine vom Handeln aktuell und zukünftig Lebender (bspw. Umweltpolitik) unabhängige individuellen Interessen in der Zukunft bestimmbar: Existenz, Anzahl und Identität zukünftig Lebender hängen von jeweils aktuellen Kontexten ab. Je nach Verlauf und Szenario entstehen also unterschiedliche Populationen (mit unterschiedlichen Menschen mit unterschiedlichen Bedürfnissen). Wer also auf so etwas wie die „Natur des Menschen“ rekurriert und damit mehr meint als nur die basalsten Bedürfnisse, die mit der jetzigen Biologie des Menschen gegeben sind (Körtner 2013, 204), der muss klarmachen, welche Gründe es gibt, so etwas zu tun. Diese Gründe scheinen mir in der theologischen Debatte fast vollständig zu fehlen.

Schon vor mehr als 50 Jahren hat sich übrigens der katholische Theologe Karl Rahner mit derartigen Fragen beschäftigt: „Der Mensch ist grundsätzlich operabel und darf es sein" (Rahner 1966, 52), so seine These. Und vor diesem Hintergrund kann das „Experiment Mensch“ (so der Titel seines Aufsatzes) natürlich scheitern. Es könnte passieren, „dass die Menschheit sich tatsächlich einmal biologisch zurückkreuzt auf die Stufe einer technisch intelligenten und selbstdomestizierten Australopithecusherde oder eines Insektenstaates ohne den Schmerz der Transzendenz ... und den Dialog mit 
Gott, also sich selbst durch kollektiven Selbstmord auslöscht" $(1966,69) .{ }^{15}$ In jüngster Zeit hat Bostrom eine analoge Dystopie wieder aufgenommen. Er diskutiert jenseits gängiger moralischer Intuitionen nicht nur die Möglichkeit der Herstellung von „emulation workers“ (Bostrom 2014, 204 und $207 \mathrm{ff}$.), sondern auch die Frage, wie sie konstruiert werden sollten, damit sie sowohl möglichst effizient arbeiten wie auch möglichst viel Glück empfinden können. Er fügt dann noch an, und damit wird meine These von der Infragestellung unserer gewohnten moralischen Überzeugungen bestätigt: „We must resist the temptation to project our own sentiments onto the imaginary emulation worker. The question is not whether you would feel happy if you had to work constantly and never again spend time with your loved ones - a terrible fate, most would agree" (Bostrom 2014, 207).

Die Frage ist natürlich, wie man mit derartigen Dystopien umgehen soll. Müssen entsprechende Möglichkeiten verhindert werden, weil ein Weiterleben der biologischen Spezies Mensch mit ihren heutigen Eigenschaften und grundlegenden moralischen Konzeptionen in sich selbst moralisch wertvoll ist?Ist es eine moralische Pflicht, gegen die befürchteten Entwicklungen anzukämpfen? Karl Rahners oben zitierte Aussagen scheinen mir stattdessen wie von einem resignativen Grundton getragen: Zwar würden wir heute Lebenden eine Welt ohne Autonomie und Transzendenzerfahrungen nicht wertschätzen wollen, aber dass eine derartige Welt keine Wirklichkeit wird, scheint nichts, was primär durch moralische Appelle adressiert werden könnte.

Vor dem Hintergrund derartiger Fragen, schlage ich vor zu prüfen, ob die eigentlichen Herausforderungen vielleicht weniger darin bestehen, noch mehr prinzipienethische Reflexion ins Spiel zu bringen, sondern eher die Geschichten und Bilder betreffen, mit denen wir unserm Leben Sinn geben, also eher psychologisch-existenzieller Art sind. ${ }^{16}$ Ich kann die damit verbun-

15 Vgl. dazu auch die aktuelle Diskussion in Zahl (2019), der der Frage nachgeht, was es im Hinblick auf das Christsein bedeuten könnte, wenn die menschlichen Affekte wie bspw. Angst vor dem Tod, vor dem Leiden, vor dem Versagen und vor Zurückweisung durch biotechnologische Interventionen unter Kontrolle gebracht würden, die Jenseitsund Erlösungshoffnungen sozusagen ihr Substrat verlieren würden.

16 Die Überlegungen von Thweat (2018) gehen m. E. in diese Richtung. Sie führt im Hinblick auf die mit der Körperlichkeit verbundene Verletzlichkeit an der Biographie von Jesus narrativ aus, wie wichtig diese im Hinblick auf Beziehungen zu Andern ist (besonders 2018, 375). Zur Frage stehe nicht eine „Unantastbarkeit des natürlichen Körpers". Im Vordergrund stehe das, was körperhaften Menschen mit ihm an Beziehungen möglich ist. 
denen Fragen hier nur andeuten. Macht ein menschliches Leben noch Sinn, ist es überhaupt noch lebenswert, wenn es keine biologischen und zeitlichen Grenzen mehr hat? ${ }^{17}$ Würden bei der Abwesenheit von Schmerzen, Leiden und Trauer und immerwährenden positiven Gefühlzuständen nicht Routine und Langweile überhandnehmen? Würde nicht der besondere Sinn von individuellen Biographien verschwinden?18 Ich kann diese Fragen hier nicht aufnehmen. Sie sollen nur deutlich machen, dass wir es nicht primär mit Fragen zu tun haben, die moralische Prinzipien und Normen betreffen, die man auf das Problem im Hinblick auf eine Entscheidung anwenden könnte. Diese kommen ins Spiel, wenn vorausgesetzt ist, dass biologisches und zeitliches Leben Sinn macht. Sie dienen dem Schutz und der Entfaltung dieses biologisch und zeitlich begrenzten, und daher vulnerablen Lebens. Die darüber hinaus gehende Frage, ob wir ein derartiges biologisch und zeitlich begrenztes, und daher vulnerables Leben führen wollen, kann mit Hinweis auf moralische Prinzipien und Normen, wie sie etwa im Falle der Menschenwürde- und Menschenrechtskonzeptionen vorliegen, nicht beantwortet werden.

\subsection{Die unscharfen Grenzen zwischen menschlichen und nicht- menschlichen Entitäten (Problem 2)}

Dem TH wird, wie gesehen, im Sinne einer Kritik gerne vorgeworfen, er wolle die Verletzbarkeit, Endlichkeit (also Geburtlichkeit und Sterblichkeit) und Körperlichkeit des Menschen überwinden. ${ }^{19}$ Als Abgrenzung gegenüber dem TH wird in der theologischen Kritik oft auf die körperlich-vergängliche Geschöpflichkeit des Menschen rekurriert (Deane-Drummond 2011; Körtner 2013). Katharina Klöcker $(2018$, 335) spricht explizit von

17 Vgl. dazu McKenny 2011, 181. Gotlind Ulshöfer hat mich darauf aufmerksam gemacht, dass Materialität selbstverständlich auch in den TH-Visionen eine Rolle spielt. Insofern werden Zerfall und Endlichkeit auch hier relevant sind. Sie würden natürlich eine andere Rolle spielen, wenn es, wie in einigen TH-Visionen vorgesehen, gelänge, so etwas wie "Geist“ von einem auf ein anderes Substrat zu laden (vgl. zum so genannten „up loading“ oder „whole brain emulation“ Bostrom 2014, 35 ff.). Damit ist natürlich auch wieder das Problem angesprochen, ob eine derartige Existenz - aus unserer Perspektive - Sinn machen würde.

18 Mehr dazu bei Loh 2019a, $46 \mathrm{f}$. im Anschluss an Hans Jonas und vor allem Hannah Arendt. Vgl. auch die neueren biokonservativen Argumente von Leon Cass, Francis Fukuyama und Michael Sandel (vgl. dazu Burdett \& Lorrimar 2019, 246 f.).

19 Zur grundsätzlichen philosophischen Analyse und Kritik vgl. Loh 2019a. 
der „gottgewollte Leiblichkeit“ des Menschen. Theologisch leuchtet diese Abgrenzung durchaus ein. ${ }^{20}$

Die am Leib festgemachte Grenzziehung gegenüber den transhumanen Wesen impliziert allerdings, dass eine andere Grenze geöffnet wird, die klassisch-biologische Grenze zu den nicht-menschlichen Tieren. Es ist kein Zufall, dass Deane-Drummond (2011, 125) in diesem Zusammenhang explizit von „human solidarity with other animals“ spricht. ${ }^{21} \mathrm{Um}$ das spezifisch Menschliche gegenüber transhumanen Wesen zu sichern, wird die in der klassischen Theologie so wichtige Grenze zu den nicht-menschlichen Geschöpfen verwischt. Es kommt hier explizit oder implizit zu einem Fokus auf die biologische Verwandtschaft mit anderen Lebewesen, die im Rahmen der natürlichen Evolution gemeinsam mit dem Menschen entstanden und gerade nicht vom Menschen gemacht worden sind. Menschen und Tiere werden theologisch als Geschöpfe Gottes (nicht des Menschen) wahrgenommen. Es sind nicht zuletzt derartige Einsichten, die den säkularen Begriff „Würde der Kreatur“, wie er in der Schweizer Bundesverfassung 120 Abs. 2 vorkommt, auch theologisch interessant machen.

Man kann diese Strategie gegen den $\mathrm{TH}$ einschlagen, muss sich dann allerdings klarmachen, dass damit Probleme verbunden sind. Der Fokus auf biologische Verwandtschaft als Basis einer Ethik ist paradigmatisch von Peter Singer mit seinem naturalistischen „expanding the circle“ (1981) artikuliert worden. Peter Singer behauptet ja bekanntlich, dass nicht biologische Speziesgrenzen (zwischen Menschen und Tieren), sondern Interessen, die an bestimmte Eigenschaften (wie bspw. Empfindungsfähigkeit oder Zeit- und Selbstbewusstsein) gekoppelt sind, moralisch relevant seien. Vor diesem Hintergrund wird die moralische Unterscheidung zwischen Menschen und nicht-menschlichen Tieren obsolet. Den höchsten Schutz genießen gemäß Singer Personen, unabhängig davon, ob es menschliche oder nicht-menschliche Personen sind (Singer 2011). Zwei Fragen müssten hier beantwortet werden.

20 Dahinter steht ja eine Idee, die Jenifer Jeanine Thweat schön zum Ausdruck gebracht hat: „Die Fleischwerdung verdeutlicht, dass nicht einmal Gott ohne einen Körper wirklich menschlich sein kann“" (Thweatt 2018, 373). Zur Kritik an diesem Rekurs auf Leiblichkeitsvorstellungen vgl. Zahl 2019.

21 Analog übrigens auch Harari: „Gerade in einer Zeit, in der wir uns in Götter verwandeln wollen, ist es doppelt wichtig, an unsere Ursprünge zu erinnern. Keine Untersuchung unserer göttlichen Zukunft kann unsere animalische Vergangenheit oder unsere Beziehungen zu anderen Tieren ignorieren“ $(2015$, 95). Dies führt beim ihm zu einer Kritik an der Art und Weise wie wir mit domestizierten Tieren umgehen (Harari 2015, $110 \mathrm{ff}$.$) .$ 
1. Wie wollen sich theologische Vertreter*innen der kreatürlichen Leiblichkeit gegen derartige Naturalisierungstendenzen in der Ethik abgrenzen, ohne die moralisch bedeutsame Verwandtschaft von Mensch und nichtmenschlicher Kreatur aufzugeben?

2. Wie wollen theologische Vertreter*innen der kreatürlichen Leiblichkeit (anders als Singer und weitere Vertreter*innen der radikalen Tierethik) eine Sonderstellung des Menschen als Ebenbild Gottes vertreten, ohne die proklamierte Solidarität als Kreatur mit anderen Kreaturen aufzugeben?

Früher oder später wird man in diesem Kontext einer naturalisierten Ethik zudem auf ein Problem stoßen, welches die diskutierten Fragen noch schwieriger machen: Gesetzt der Fall, Roboter oder AI-Systeme lassen sich bezüglich bestimmter Fähigkeiten und ihrem funktionalen Innenleben doch nicht mehr so klar von biologischen Organismen wie Tieren oder Menschen unterscheiden, wie der theologische Fokus auf Leiblichkeit suggeriert. Wäre es dann nicht eine ethische unzulässige Diskriminierung, wenn man sie anders als Menschen oder empfindungsfähige Tiere behandelt?22 Will man derartigen nicht-menschlichen Wesen einen moralischen Status nur deswegen nicht zusprechen, weil sie keine dem Menschen (oder bestimmten Tieren) vergleichbare biologisch fundierte Leiblichkeit haben? Was, so müssen sich zudem Theolog*innen fragen, verbietet es, TH/AI-Entitäten als Geschöpfe Gottes wahrzunehmen? Einfach das definitorische Vorurteil, was nicht auf die Weise menschlich sei, wie wir es kennen, dürfe es auch nicht sein? Was, wenn nicht ein biologistisches Vorurteil, spricht dagegen als „meinen Nächsten“ (im Sinne der Bibel) jedes Wesen wahrzunehmen, mit dem bestimmte Formen der Kommunikation und Gemeinschaft möglich sind (vgl. Campell \& Garner 2016, 88), also auch trans- oder posthumane Wesen? Unterdessen mehren sich ja die empirischen Befunde, dass Menschen durchaus mit IT/AI-basierten Systemen kommunizieren können. Unterschiedliche Chatbots übernehmen zunehmend Funktionen im Leben von Menschen. ${ }^{23}$ Hier findet virtuelle Kommunikation statt, die sehr real

22 So Bostrom \& Yudkowsky 2015 mit ihrem "Principle of Substrate Non-Discrimination”. Vgl. auch den Überblick über diese Debatte bei Loh 2019b, 72-95.

23 Vgl. etwa den bekannt gewordenen Chatbot von Eugenia Kuyda. Dabei handelt es sich um ein künstliches neuronales Netzwerk, das aufgrund der Emails, die sie von ihm hat, so chattet, wie ihr verstorbener Freund Roman Mazurenko. Sie hat daraus den Chatbot „Replika“ entwickelt (https://replika.ai/), dem 2019 schon über 100.000 Menschen ihre Sorge und Ängste anvertrauen (so https://www.gdi.ch/de/publikationen/trend-updates/e in-chatbot-fuer-schwere-stunden, Zugriff am 24.7.2019). 
ist. Was ist der Unterschied zur Kommunikation mit einem ja auch nicht empirischen Gegenüber wie Gott? Ich halte derartige Fragen für enorm spannend im Hinblick auf unser Verständnis von kommunikativer Wirklichkeit. Gibt es einen nachvollziehbaren Test, der es erlauben würde, die Kommunikation mit Gott eindeutig von der Kommunikation mit einem Chatbot abzugrenzen? ${ }^{24}$

\subsection{Die Grenzen des technologischen Fortschrittes (Problem 3)}

Mit wenigen Ausnahmen hält die Theologie Technologie und Wandel durch Technologie für moralisch legitim. Paradigmatisch etwa Klöcker im Anschluss an Rahner: „Gerade in diesem Auftrag der Gestaltung des Vorgegebenen, der Natur, liegt das den Menschen Auszeichnende und ihn mit Gott Verbindende" (Klöcker 2018, 325 ff., analog Campbell \& Garner 2016, 142). An dieser positiven Beurteilung von Technologie wird festgehalten, solange jedenfalls „proper limits of technological progress" respektiert werden (Peters 2011, 72). So gelten bspw. Enhancement-Projekte gemäß Ted Peters als erlaubt, wenn deren Ziele „human health and wellbeing" verbessern (Peters 2011, 82; analog Campbell \& Garner 2016, 142) oder wenn sie sich im Hinblick auf die "Wahrung von Autonomie“ in ihren Folgen als „nicht-kontraproduktiv“ erweisen (Klöcker 2018, 330).

Die entscheidende Frage bleibt natürlich: Wo genau würde die Grenzüberschreitung stattfinden? Meine Diskussion in Kap. 2.1. hat gezeigt, dass mit der möglichen Antwort „Wenn die Bedingungen von menschlicher Autonomie zerstört werden" erhebliche Probleme verbunden sind. Im Hinblick auf menschliche Gesundheit und Wohlergehen sind nüchterne Kosten-Nutzen-Abwägungen erforderlich, wie sie auch in der Theologie unbestritten sind. Dennoch kann ich mich des Eindrucks nicht erwehren, dass die Technologien, die im Zusammenhang mit TH/AI diskutiert werden, von Seiten der Theologie gerne grundsätzlich als Ausdruck menschlicher Hybris, Sünde oder gar des Dämonischen interpretiert werden (vgl. Peters 2011; Childs 2015). Dazu eine m. E. typische Aussage:

„Vorsicht ist geboten im Blick auf die Beteuerung, es handle sich bei Human Enhancement nur um eine Steigerung der Heilungsmöglichkeiten des Menschen. Die Tradition und Gegenwart zeigt, dass der Ausgriff höher reicht und mit Visionen und Utopien eines anderen, neuen gottähnlichen Menschen spielt. Die technischen Entwicklungsmöglichkeiten werden dabei durchaus befürwortet, ohne jedoch in unkontrollierten Machbarkeitswahn zu verfallen. Zu Recht wird in Erinnerung

24 Es würde sich hierbei um eine Art theologischen Turing-Tests handeln. 
an die Bedeutung der Leiblichkeit des Menschen ein Panorama zur Reflexion des Menschseins im 21. Jh. aufgespannt. Dieses kann dazu dienen, die transhumanistische Vorstellung des neuen Maschinen-Menschen durch die Erinnerung an dessen Mitsein und Mitfühlen mit anderen und nicht zuletzt seine Geburtlichkeit zugunsten eines Humanismus des freien Menschen zu erden." (Gräb-Schmidt 2012, 232)

Der Rekurs auf bestimmte Attribute des Menschen (Autonomie, Leiblichkeit) und menschlichen Zusammensein (Mitfühlen) ist zwar verständlich. Aber mit diesem Rekurs allein sind noch in keinerlei Weise normative Grenzen gegeben. Vielleicht wäre es an der Zeit gemeinsam mit Kevin Kelly, dem Gründer des „Wired“-Magazins, folgende Wette einzugehen: „We tend to see God reflected in nature, but my bet is that technology is the better mirror of god" (Kelly 1999, 392).

\section{Fazit und Ausblick}

Zweifelsfrei steht die Theologie angesichts von TH/AI vor großen Herausforderungen. Was bedeuten Begriffe wie „Kreatürlichkeit“, „Gabe des Lebens“, „Ebenbild Gottes“, „Gnade und Erlösung“ usw. in diesem Kontext?25 Mit welchen Gründen und mit welcher Sprache kann diese theologische Begrifflichkeit ins Spiel gebracht werden, ohne die neuen technologischen Projekte und Möglichkeiten bloß zu verurteilen? Derartige Fragen haben sich bei jeder neuen sozialen Institution und Technologie gestellt, welche die Herrschaftsmacht des Menschen ausdehnte. Eine bekannte theologische Strategie schlägt vor, dass es zur Aufgabe menschlichen Lebens gehört, als Stellvertreter oder Mandatar Gottes in die Schöpfung einzugreifen und diese sozusagen gemeinsam mit Gott weiter zu entwickeln. ${ }^{26}$

25 Einen interessanten Versuch macht Kathryn Tanner 2009, die wie die TH von der radikalen Gestaltbarkeit („malleability“) menschlicher Wesen ausgeht. Sie lehnt “a stable, fixed, and clearly demarcated human nature” ab (2009, 363 f.) Aber diese Gestaltbarkeit oder Plastizität ist kein Selbstzweck: "It is not just plasticity, then, that is at issue when considering humans as the image of God, but plasticity of a certain sort: openness to, essential dependence upon, the influence of the alien or foreign - God, who is other than creatures, what they are not" (2009, 371). Diese Ausführungen haben eine gewisse Nähe zur der von Loh 2019a als „kritischer Posthumanismus“ bezeichneten Position.

26 Vgl. dazu Hefner 1993. Auch bei ihm definieren allerdings die Ziele Gottes mit der Natur die Grenzen der menschlichen Freiheit (Hefner 1993, 39 ff.). 
Aber die erwähnten Fragen scheinen sich diesmal mit besonderer Dringlichkeit zu stellen. ${ }^{27}$

Ich sehe drei Möglichkeiten, welche die entsprechenden Debatten von Seiten der Theologie her weniger steril und defensiv machen und damit verbessern könnten. Ich schlage eine Diskussion vor mit

1. einem Rückgriff auf die Thematik der Kontingenz;

2. der Aufnahme der transformatorischen Energie, wie sie die TH/AIVisionen umgibt;

3. einem positiven Fokus auf Fragen der Selbstgestaltung der Menschen.

Zum ersten Punkt: Auch wenn die technologischen Möglichkeiten sehr viel eingriffsmächtiger sind als heute, wird die Umwelt der transhumanen Wesen nicht perfekt sein. Unverfügbares und Kontingentes wird auch dort begegnen (Coeckelberg 2018, 87; Körtner 2013, 208; Klöcker 2018, 333). Zu Recht moniert Ted Peters (2011, 78 ff.) die diesbezügliche Naivität der TH. Es ist wohl kein Zufall, dass Hartmut Rosa in seinem Buch über Unverfügbarkeit (2018) von „der Rückkehr des Unverfügbaren als Monster" (2018, 116 ff.) spricht, dem wir heute gerade in den Kontexten avanciertester Verfügbarkeitstechniken begegnen. Allerdings liest sich gerade Nick Bostroms (2014) umfassende und teilweise hoch spekulative Analyse im Wesentlichen als ein ernüchterter und sehr ernüchternder Versuch, die möglichen Folgen einer TH/AI-Zukunft zu denken Strategien zu entwickeln, die die möglichen Ereignisse und Entwicklungen doch irgendwie unter Kontrolle bringen. Von Seiten der Theologie her wird man fragen dürfen, ob wir in diesen Kontexten nur Monstern und Dämonen als Alpträumen begegnen werden, oder ob auch Entwicklungen und Begegnungen stattfinden werden, in denen Liebe, Vergebung und Gnade Thema sind. Wenn wir davon ausgehen müssen, dass Kontingenzen nicht generell beseitigt werden können, braucht es neben den TH/AI-Utopien angepasste Strategien der Kontingenzbewältigung (vgl. Klöcker 2018, 333). Vorausgesetzt natürlich die trans- oder posthumanen Wesen haben entsprechende existenzielle Interessen. Welche positive Rolle könnten religiöse und theologische Narrative, Semantiken der Symbole dabei spielen? Diese Frage ist nicht zuletzt deswegen interessant, weil der $\mathrm{TH}$ ja mit Visionen operiert, deren Nähe zu christlichen Themen des ewigen Lebens, des Lebens in Fülle usw. nicht zu übersehen ist (Coeckelberg 2018; Thweatt 2018, 368 ff.).

27 Mehr denn je scheinen insbesondere die biblischen Metaphern und Narrative, die ja im Wesentlichen dem Bereich der Landwirtschaft und der Politik entstammen (Campbell \& Garner 2016, 91), hier an ihre Grenzen zu stoßen. 
Zum zweiten Punkt: Als einer der wenigen deutschsprachigen Theologen versucht Mathias Wirth positiv an die TH/AI-Visionen anzuknüpfen. ${ }^{28}$ So hebt er (2018, 162 ff.) Parallelen zwischen AI/TH und Theologie hervor. In beiden Fällen gehe es um die Hoffnung auf einen „neuen Menschen“ und „Überwindung des Todes“. Die Vertreter von TH/AI sind diesbezüglich auf eine Weise optimistisch, wie es Theologen*innen kaum je sind. Und insofern haben sie „rebellisches Potential“ gegenüber der Welt, wie sie ist (Wirth 2018, 151). Wirth formuliert den Punkt auf diese Weise und macht damit deutlich, dass man TH/AI-Visionen nicht als eskapistisch-gnostische Orientierung interpretieren muss: „Es steht nicht fest, dass der Glaube an einen von Gott geschaffenen neuen Himmel und eine neue Erde im Widerspruch zu Visionen und Konzepten einer signifikanten Steigerung menschlicher Fähigkeiten durch medizinische und technische Einflussnahme steht" (Wirth 2018, 151). Mit Sätzen dieser Art wirkt er der Gefahr der Vertröstung und Verschiebung auf eine bloß gedachte „Zukunft bei Gott“ (Wirth 2018, 153) entgegen. Natürlich ist hier die theologische Kritik schnell zur Hand, es gehe im Falle christlicher Eschatologie um den „Advent Gottes“ („divine transformation“) statt ein blosses „Futurum des Menschen“ (Peters 2011, 74). ${ }^{29}$ Aber müsste, wer eine futurische Eschatologie vermeiden will, nicht auch im von Menschen Gemachten und Geplanten den Advent Gottes wahrnehmen können?

Zum dritten und letzten Punkt: Janina Loh hat in ihrer Einführung zum Trans- und Posthumanismus die Übergänge vom klassischen Humanismus zu den neueren Entwicklungen des Transhumanismus schön herausgearbeitet. Was demr aufmerksamen Leser*in auffällt: Im von ihr als Kontrastfolie herbeigezogenen Humanismus der Tradition spielt die Selbstgestaltung und Kultivierung der Person (im Anschluss an antike Lebensführungstechniken) eine entscheidende Rolle (Loh 2019a, 20 und 69). Der $\mathrm{TH}$ steht in dieser Tradition, es kommt aber zu einem entscheidenden Unterschied. Nicht die Selbstgestaltung durch „aus dem Selbst generierte“, wenn man will „psychologische“ oder "interne“ Techniken steht im Vordergrund. Angestrebt ist vielmehr eine Transformation des Menschen durch „äußere“ Techniken und Hilfsmittel (Computer- und Gentechnologie,

28 Vgl. daneben auch Zahl 2019, der „biotechnological enhancement“, allerdings in bestimmten Grenzen, als „means of grace“ $(2019$, 223) diskutiert. Seine Strategie besteht schließlich darin, eine zu nahe Verbindung zwischen „theological realities before God" und den an den Körper gebundenen Affekten zu hinterfragen (Zahl 2019, 226).

29 Analog auch Körtner mit seiner theologischen These, dass die finale Bestimmung des Menschen etwas sei „which he himself cannot achieve“ $(2013,209)$. 
Pharmakologie usw.). ${ }^{30}$ Die Transformierung des Menschen erfolgt über eine naturwissenschaftliche Objektivierung dessen, was er als biologisches Wesen ist, damit er - sowie man das im Rahmen von Technik gewohnt ist technisch umgeformt werden kann. ${ }^{31}$

Was hat das mit der Theologie zu tun? Theologische Ansätze setzen mit ihrer Kritik an der Selbsttransformation des Menschen in der Regel sehr grundsätzlich an. Sie verurteilen vor dem Hintergrund der Kreativität Gottes kreative Innovationen des Menschen an sich selbst gerne als Anmaßung. Die bspw. von Ulrich Körtner (2013, 209) stark gemachte alleinige Aktivität Gottes im Hinblick auf die Vollendung des Menschen ist zwar theologisch nachvollziehbar, nicht zuletzt wenn es um Fragen wie das ewige Leben oder Erlösung geht. Ich frage mich aber, ob mit diesem vor allem protestantischen Fokus auf die Kreativität Gottes nicht auch ein möglicher Punkt in der Debatte mit dem TH verspielt wird? Ließe sich das Anliegen des TH nicht besser und kritischer diskutieren, wenn man die individuelle Selbstgestaltung nicht zu grundsätzlich in Frage stellt? Käme theologischerseits in dieser Debatte nicht weiter, wer im Hinblick auf die Selbsttransformation des Menschen nicht zu früh Selbsterlösung wittert? Auch ein theologisches Verständnis einer Kultur des Umgangs mit dem Unverfügbaren muss neben der Rezeptivität des Menschen (vor Gott), dessen kreative Selbstgestaltung denken können - wenn sie denn für Menschen verständlich und lebbar sein will ${ }^{32}$.

30 Vgl. insbesondere zur Frage der Kontrolle unserer Affekte die Diskussion von Zahl 2019. Auf der Basis einer anthropologischen Metaphysik, deren Gehalt ich hier nicht diskutieren will, analysiert Grössl (2018) die von ihm so genannten „character forming enhancements“ durchaus ergebnisoffen. Allerdings heißt es dann auch bei ihm: „Bei der Verwendung von neuen Technologien zur Unterstützung der Charakterentwicklung muss aber immer bedacht werden, dass diese weder die eigene Anstrengung noch Gottes Unterstützung ersetzen könne" (Grössl 2018, 359).

31 Vgl. zu der damit implizierten Differenz von Selbst und Körper sowie eine damit verbundene Kritik Jennifer Jeanine Thweatt in ihrem Text zur „Heiligkeit des Körpers“ (2018, 366 f. und 375).

32 Vgl. dazu die schon etwas älteren Texte von Josuttis (2002) und Huppenbauer (2008), sowie neuerdings Rosa (2018) mit der Verkoppelung von Resonanz und Selbstwirksamkeit. Burdett \& Lorrimar (2019) nehmen das Verschränktsein von menschlicher Rezeptivität und Gestaltungskraft im Anschluss an Kathryn Tanner auf. 


\section{Literaturverzeichnis}

Birnbacher, Dieter. 2006. Natürlichkeit. Berlin u. New York: de Gruyter. Bostrom, Nick. 2014. Superintelligence. Paths, Dangers, Strategies. Oxford: Oxford University Press.

Bostrom, Nick and Yudkowsky, Eliezer. 2014. "The ethics of artificial intelligence." In The Cambridge Handbook of Artificial Intelligence, ed. by Keith Frankish, 316-334. Cambridge: University Press.

Burdett, Michael and Lorrimar, Victoria. 2019. "Creatures Bound for Glory: Biotechnological Enhancement and Visions of Human Flourishing." Studies in Christian Ethics 32(2): 241-253.

Campbell, Heidi A. and Garner, Stephen. 2016. Networked Theology. Negotiating Faith in Digital Culture. Grand Rapids: Baker Academic.

Childs, James M. 2015. "Beyond the Boundaries of Current Human Nature: Some Theological and Ethical Reflections on Transhumanism." In Dialog: A Journal of theology, 54(1): 8-19.

Coeckelberg, Mark. 2018. „Transzendenzmaschinen: Der Transhumanismus und seine (technisch-) religiösen Quellen." In Designobjekt Mensch. Die Agenda des Transhumanismus auf dem Prüfstand, hrg. v. Göcke, Benedikt Paul und Meier-Hamidi, 81-113. Freiburg: Herder.

Cole-Turner, Ronald, ed. 2011. Transhumanism and Transcendence. Christian Hope in an Age of Technological Enhancement. Washington: Georgetown University Press.

Deane Drummond, Celia. 2011. "Taking Leave of Animals? The Theological and Ethical Implications of Transhuman Projects." In Transhumanism and Transcendence. Christian Hope in an Age of Technological Enhancement, ed. by Cole-Turner, Ronald, 115-130. Washington: Georgetown University Press.

Göcke, Benedikt Paul und Meier-Hamidi, Frank. Hrg. 2018. Designobjekt Mensch. Die Agenda des Transhumanismus auf dem Prüfstand. Freiburg: Herder.

Gräb-Schmidt, Elisabeth. 2012. „Die Debatte über 'Human Enhancement': Historische, philosophische und ethische Aspekte der technologischen Verbesserung des Menschen." In Theologische Literaturzeitung 137(2): 230-232.

Grössl, Johannes. 2018. „Verbesserung oder Zerstörung der menschlichen Natur? Eine theologische Evaluation des Transhumanismus." In: Designobjekt Mensch. Die Agenda des Transhumanismus auf dem Prüfstand, 
hrg. v. Göcke, Benedikt Paul und Meier-Hamidi, 339-361. Freiburg: Herder.

Habermas, Jürgen. 2001. Die Zukunft der menschlichen Natur: Auf dem Weg zu einer liberalen Eugenik? Frankfurt: Suhrkamp.

Hefner, Philip. 1993. The Human Factor: Evolution, Culture, Religion. Minneapolis: Fortress Press.

Heilinger, Jan. 2016. Grenzen des Menschen. Zu einer Ethik des Enhancement, Bundeszentrale für politische Bildung. Zugriff 3.7.2019. https://ww w.bpb.de/apuz/233466/grenzen-des-menschen-zu-einer-ethik-des-enhan cement.

Huppenbauer, Markus. 2008. „Handeln im Kontext von Unverfügbarkeit. Probleme des Wissenstransfers zwischen Management und Theologie." In Management und Spiritualität. epd Dokumentation 44/45: 33-42. Frankfurt.

Josuttis, Manfred. 2002. Religion als Handwerk. Zur Handlungslogik spiritueller Methoden. Gütersloh Gütersloher Verlag-Haus.

Kelly, Kevin. 1999. "Nerd theology." In: Technology in Science 21(4): 387392.

Klaes, Christian. 2018. „Was steht hinter den Versprechen des Transhumanismus? Eine naturwissenschaftliche Perspektive." In Designobjekt Mensch. Die Agenda des Transhumanismus auf dem Prüfstand, hrg. v. Göcke, Benedikt Paul und Meier-Hamidi, 379-408. Freiburg: Herder.

Klöcker, Katharina. 2018. „Zur ethischen Diskussion um Enhancement.“ In Designobjekt Mensch. Die Agenda des Transhumanismus auf dem Prüfstand, hrg. v. Göcke, Benedikt Paul und Meier-Hamidi, 308-338. Freiburg: Herder.

Kirchschläger, Peter G. 2015. „Der bewusste Verzicht der Menschenrechte auf ein Menschenbild - Über die Legitimation der Rede über ein Menschenbild der Menschenrechte." In Natur des Menschen. Brauchen die Menschenrechte ein Menschenbild, hrg. v. Bogner, Daniel und Mügge, Cornelia, 133-143. Fribourg: Academic Press Fribourg.

Körtner, Ulrich. 2013. "The Enhanced Human? Ethical Assessments and Implicit Images of Humanhood from a Theological Perspective." In: Human Enhancement: Scientific, Ethical and Theological Aspects from a European Perspective, ed. by Boer, Theo and Fischer, Richard, 193-215. Strasbourg.

Kurzweil, Raymond. 2005. The Singularity is near. When humans transcend biology, New York: Viking. 
Loh, Janina. 2019a. Trans- und Posthumanismus zur Einführung, 2. überarbeitete Aufl. Hamburg: Junius.

Loh, Janina. 2019b. Roboterethik. Eine Einführung. Frankfurt: Suhrkamp (stw).

McKenny, Gerald. 2011. "Transcendence, Technological Enhancement, and Christian Theology." In 2011. Transhumanism and Transcendence. Christian Hope in an Age of Technological Enhancement, ed. by Cole-Turner, Ronald, 177-192. Washington: Georgetown University Press.

More, Max. 1990. Transhumanism. Towards a Futurist Philosophy. Zugriff 25.7.2019. https://web.archive.org/web/20110216221306/http:/www.m axmore.com/transhum.htm.

Parfit, Derek. 1987. Reasons and Persons, Oxford: Oxford University Press. Peters, Ted. 2011. "Progress and Provolution. Will Transhumanism Leave Sin Behind?" In Cole-Turner, Ronald, ed. 2011. Transhumanism and Transcendence. Christian Hope in an Age of Technological Enhancement, 63-86. Washington: Georgetown University Press.

Rosa, Hartmut. (2018). Unverfügbarkeit. Salzburg: Residenz.

Singer, Peter. 1981. The Expanding Circle. Ethics, Evolution, and Moral Progress. Princeton: Princeton University Press.

Peter Singer. 2011. Practical Ethics, 3. ed. Cambridge: Cambridge University Press.

Sloterdijk, Peter. 1999. Regeln für den Menschenpark. Ein Antwortschreiben zu Heideggers Brief über den Humanismus, Suhrkamp, Frankfurt: Suhrkamp.

Tanner, Kathryn. 2009. "Grace without Nature." In Without Nature? A New Condition for Theology, ed. by Albertson, David and King, Cabell, 363375. New York: Fordham University Press

Thweatt, Jennifer Jeanine. 2018. „Cyborg Christus: Transhumanismus und die Heiligkeit des Körpers." In Designobjekt Mensch. Die Agenda des Transhumanismus auf dem Prüfstand, hrg. v. Göcke, Benedikt Paul und Meier-Hamidi, 363-376. Freiburg: Herder.

Weir, Ralph Stefan. 2018. „Transhumanismus und die Metaphysik der menschlichen Person." In Designobjekt Mensch. Die Agenda des Transhumanismus auf dem Prüfstand, hrg. v. Göcke, Benedikt Paul und MeierHamidi, 225-258. Freiburg: Herder.

Wirth, Mathias. 2018. „Doketisch, pelaganisch, sarkisch? Transhumanismus und technologische Modifikationen des Körpers in theologischer Perspektive." Neue Zeitschrift für systematische Theologie 60(1): 142-167. 
Rahner, Karl. 1966. „Experiment Mensch. Theologisches über die Selbstmanipulation des Menschen." In Die Frage nach dem Menschen. Aufriss einer philosophischen Anthropologie, hrg. von Rombach, Heinrich Rombach, 45-69. Festschrift für Max Müller zum 60. Geburtstag, Freiburg und München: Alber.

Spieckermann, Sarah. 2019. Digitale Ethik. Ein Wertesystem für das 21. Jahrhundert. München: Droemer \& Knaur.

Zahl, Simeon. 2019. "Engineering Desire. Biotechnological Enhancement as Theological Problem.” Studies in Christian Ethics 32(2): 216-228.

Zimmermann, Markus. 2015. „Grenzverschiebungen - Zur Natur des Menschen in bioethischen Diskursen." In Natur des Menschen. Brauchen die Menschenrechte ein Menschenbild, hrg. v. Bogner, Daniel und Mügge, Cornelia, 175-195. Fribourg: Academic Press Fribourg. 